\title{
Endoscopic Papillary Balloon Dilation Versus Sphincterotomy for Removal of Small Common Bile Duct Stones in Young Patients: Unresolved Issues for an Expanding Technique
}

\author{
Imad Elkhatib • Thomas J. Savides
}

Published online: 22 February 2014

(c) Springer Science+Business Media New York 2014

Endoscopic papillary balloon dilation (EPBD) to assist removal of common bile duct (CBD) stones was introduced as an alternative to endoscopic sphincterotomy (ES) under the theory that by avoiding permanent ablation of the sphincter of Oddi, long-term morbidity may be reduced. After ES, the function of the biliary sphincter is permanently lost, as determined by manometric studies up to 17 years post-sphincterotomy [1]. Moreover, high levels of bacterial colonization with associated deconjugation of hydrophobic bile salts and chronic common hepatic duct inflammation, possibly due to the due to reflux of luminal contents with fibrosis, occurs in patients following ES [2].

Since biliary sphincter function is not affected by EPBD, the incidence of post-procedural CBD stone formation appears to be markedly reduced compared with ES. In a study of 94 patients who had an ES 15 years prior followed by early cholecystectomy for choledocholithiasis, $\sim 11 \%$ developed recurrent CBD stones [3]. Another retrospective study reported the rate of recurrent $\mathrm{CBD}$ stone formation to be significantly lower in the EPBD group than in the ES group (4.4 vs. $12.7 \% ; P=0.048$ ) [4]. Other potential advantages of EPBD include reduced risk of post-sphincterotomy bleeding (especially in the setting of coagulopathy) and lower risk of post-endoscopic retrograde cholangiopancreatograpy (ERCP) cholecystitis, and greater ease and safety in patients with distorted anatomy such as prior Billroth II anastomosis or juxtapapillary diverticula. In a randomized, controlled trial of ES versus EPBD in 218 consecutive patients undergoing ERCP for bile duct stones, $91 \%$ of the ES patients and

I. Elkhatib - T. J. Savides $(\bowtie)$

Division of Gastroenterology, University of California, San

Diego, La Jolla, CA, USA

e-mail: tsavides@ucsd.edu
$89 \%$ of the EPBD patients had complete clearance of their stones after a single session [5]. Mechanical lithotripsy was required more often in the EPBD group (31 procedures versus 13 in the EPBD and ES groups, respectively). For patients with stones $>10 \mathrm{~mm}$ or multiple stones, however, lithotripsy requirements in the EPBD group were up to $50 \%$, with additional ES or repeat ERCP needed in 15-30 \%. Thus, while overall efficacy was comparable between ES and EPBD, the greatest practical benefit may be seen in patients with smaller $(<10 \mathrm{~mm})$ stones.

The initial enthusiasm for balloon sphincteroplasty in the 1990s was tempered by large randomized controlled studies reporting an increased risk of post-ERCP pancreatitis and morbidity. A multicenter, randomized, controlled study of 117 patients assigned to dilation and 120 to ES for choledocholithiasis was terminated at the first interim analysis due to a significantly higher rate of serious shortterm complications, including 2 deaths due to pancreatitis in the EPBD group versus the ES group [6]. The rate of pancreatitis was $15 \%$ in the EPBD group, compared to just $0.8 \%$ in the ES group $(P<0.001)$. The authors concluded that EPBD should be avoided in routine clinical practice. This and similar studies inform the current American Society for Gastrointestinal Endoscopy (ASGE) guideline statement that EPBD be considered mainly in patients with bleeding diatheses or in those with distorted anatomy that renders sphincterotomy dangerous or unfeasible, such as patients with peri-ampullary diverticula or with Billroth II anatomy [7].

In the last decade, interest in balloon sphincteroplasty has increased again with the adoption of widespread use of large balloon sphincter dilation after small sphincterotomy, which minimizes risks of perforation or pancreatitis while facilitating the removal of large stones. In this technique, a small sphincterotomy is performed followed by balloon 
dilation to $12-20 \mathrm{~mm}$. While the long-term consequences of this technique such as recurrent choledocholithiasis remain undefined, the initial experience showing lower risk of pancreatitis has made it an attractive option in selected patients for removal of large CBD stones.

In this issue of Digestive Diseases and Sciences, Seo et al. [9] report a randomized controlled trial comparing EPBD to sphincterotomy in young patients with small CBD stones. The rationale is that these young patients ( $<40$ years old) may have a higher lifetime risk of recurrent CBD stones. This is a well-designed single-center study; entry criteria include age $<40$ years, CBD diameter $\geq 6 \mathrm{~mm}$, and CBD stone diameter $\leq 12 \mathrm{~mm}$. Balloon dilation was standardized: the balloon was slowly inflated to 6-10 $\mathrm{mm}$ until the balloon waist disappeared, with expansion maintained for 90-120 s. EPBD and ES removed $\mathrm{CBD}$ stones in $>98 \%$ of cases. The need for supplementary mechanical lithotripsy was similar in both groups (8-9 \%). There were no significant differences in complications ( $8 \%$ EPBD vs. $11 \%$ ES), each with 4 episodes of mild pancreatitis and 1 episode of moderate pancreatitis, although the ES group also had two cases of bleeding and one perforation. The recurrence rate of CBD stones over a mean of nearly 3 years of follow-up was $1.6 \%$ in the EPBD group compared to $5.8 \%$ in the ES group, which was not statistically significant.

The main problem with this study is that it was not sufficiently powered to detect a statistically significant difference in morbidity for the expected calculated sample size, which was supported by the Disario study. The Disario study statistical analysis estimated that 355 patients would be needed in each group (710 patients total) in order to detect a difference in 30-day morbidity of 8 percentage points above and 5 percentage points below a $10 \%$ predicted value for sphincterotomy complications, but that study was stopped after the first interim data analysis (237 patients total) due to significantly more pancreatitis in the ES group as well as 2 deaths [6].

The mechanism for post-ERCP pancreatitis following EPBD is unclear, but has been suggested to be related to edema and spasm from the trauma of dilation, which can obstruct the pancreatic duct, causing pancreatitis. An important risk factor of EPBD for pancreatitis is the duration of sphincteroplasty balloon dilation, as reported in a meta-analysis in which the authors reported the risks of long ( $>1 \mathrm{~min})$ or short ( $<1 \mathrm{~min})$ EPBD duration vs. ES in regards to the risk of post-ERCP pancreatitis [8]. Shortduration EPBD had a significantly higher risk of pancreatitis (OR 4.1, CI 1.58-12.56) compared to ES, whereas no difference in the rate of pancreatitis (OR 1.07, CI 0.38-2.76) was reported when long-duration EPBD was compared to ES. Moreover, due to the lower risks of bleeding and perforation that occur with EPDB, the overall
Table 1 Factors that may improve safety of endoscopic papillary balloon dilation for removal of small common bile duct stones

Use MRCP or EUS to determine which patients have CBD stones

Wire -guided ERCP cannulation to avoid pancreatic duct injections

Stone size $<12 \mathrm{~mm}$

Distal bile duct $>6 \mathrm{~mm}$

Balloon size no greater than size of bile duct

Balloon dilation time $>60 \mathrm{~s}$

Administer rectal indomethacin during procedure

rate of complications was lower with long-duration EPBD than with ES (OR 0.54, CI 0.20-1.36). Many of the earlier $\mathrm{RCT}$ s that reported atypically high rates of pancreatitis following EPBD, including the Disario study, used shortduration sphincteroplasty. The authors of the current study used dilation times in excess of $1 \mathrm{~min}$ (90-120 s), which may partly explain their lower rates of pancreatitis.

The risk of pancreatitis may be related to other modifiable factors that occur during stone extraction rather from balloon dilation alone. Seo et al. [9] previously published a retrospective comparison of retrograde EPBD versus anterograde percutaneous transhepatic papillary balloon dilation (PTPBD) done by interventional radiology for stone extraction. They reported a significant difference in the rate of pancreatitis between the two groups, with pancreatitis occurring in $14(6.7 \%)$ of 208 patients in the EPBD group $($ mild $=9$; moderate $=4$; severe $=1$ ) compared to no cases of pancreatitis among the 56 patients in the PTPBD group $(P<0.05)$ [9]. From these data, the authors speculate that the process of stone extraction involving papillary trauma from cannulation, pancreatic duct cannulation, and mechanical lithotripsy may contribute to the risk of pancreatitis.

It is possible that proper patient selection and better overall ERCP techniques to prevent post-ERCP pancreatitis will help reduce the potential risks from balloon dilation for small CBD stones (Table 1). Specifically, MRCP and EUS can limit the use of ERCP to patients with definite CBD stones. Wire-guided selective cannulation and avoidance of pancreatography may also decrease overall pancreatitis rates, as may specifically performing only long-duration balloon sphincteroplasty ( $>1 \mathrm{~min}$ ). Finally, medical therapy to decrease post-ERCP pancreatitis is now commonly used, such as rectal indomethacin in patients at high risk for pancreatitis, of possible benefit to this group of young patients with small stones undergoing EPBD alone [10]. Regardless of which standardized technique is compared to biliary sphincterotomy, a large, welldesigned, randomized, controlled study with sufficient enrollment is needed to adequately identify pancreatitis risks. 
In summary, although the study by Seo et al. [9] did not report a significant difference in the complication rate between EPBD and ES in this young group of patients with small stones, the study was underpowered to detect small differences. While EPBD to remove small CBD stones may be considered in selected patients, it is important to remember that earlier randomized studies reported severe risk of pancreatitis with this technique, and therefore, clinicians using EPBD should also consider new technical and pharmacologic means of minimizing the risk of EPBDinduced pancreatitis.

\section{References}

1. Minami A, Nakatsu T, Uchida N, et al. Papillary dilation vs sphincterotomy in endoscopic removal of bile duct stones. A randomized trial with manometric function. Dig Dis Sci. 1995;40:2550-2554.

2. Bergman JJ, van Berkel AM, Groen AK, et al. Biliary manometry, bacterial characteristics, bile composition, and histologic changes fifteen to seventeen years after endoscopic sphincterotomy. Gastrointest Endosc. 1997;45:400-405.

3. Bergman JJ, van der Mey S, Rauws EA, et al. Long-term followup after endoscopic sphincterotomy for bile duct stones in patients younger than 60 years of age. Gastrointest Endosc. 1996;44:643-649.

4. Natsui M, Saito Y, Abe S, et al. Long-term outcomes of endoscopic papillary balloon dilation and endoscopic sphincterotomy for bile duct stones. Dig Endosc. 2013;25:313-321.

5. Bergman JJ, Rauws EA, Fockens P, et al. Randomised trial of endoscopic balloon dilation versus endoscopic sphincterotomy for removal of bileduct stones. Lancet. 1997;349:1124-1129.

6. Disario JA, Freeman ML, Bjorkman DJ, et al. Endoscopic balloon dilation compared with sphincterotomy for extraction of bile duct stones. Gastroenterology. 2004;127:1291-1299.

7. ASGE Standards of Practice Committee, Maple JT, Ikenberry SO, Anderson MA, Appalaneni V, Decker GA, et al. The role of endoscopy in the management of choledocholithiasis. Gastrointest Endosc. 2011;74:731-744.

8. Liao WC, Tu YK, Wu MS, et al. Balloon dilation with adequate duration is safer than sphincterotomy for extracting bile duct stones: a systematic review and meta-analyses. Clin Gastroenterol Hepatol. 2012;10:1101-1109.

9. Seo YR, Moon JH, Choi HJ, et al. Comparison of endoscopic papillary balloon dilation and sphincterotomy in young patients with CBD stones and gallstones. Dig Dis Sci. (Epub ahead of print). doi:10.1007/s10620-013-2949-6.

10. Elmunzer BJ, Scheiman JM, Lehman GA, et al. A randomized trial of rectal indomethacin to prevent post-ERCP pancreatitis. $N$ Engl J Med. 2012;366:1414-1422. 